\title{
Inovação e colaboração governamental para recuperar veículos roubados e furtados
}

Viana Guimarães, Arthur; Cabral, Sandro; Fernandes Ribeiro, Priscila; Marchesini da Costa, Marcelo Inovação e colaboração governamental para recuperar veículos roubados e furtados

Administração Pública e Gestão Social, vol. 13, núm. 4, 2021

Universidade Federal de Viçosa, Brasil

Disponible en: https://www.redalyc.org/articulo.oa?id=351568433012

\section{(c) (1) $(9)$}

Esta obra está bajo una Licencia Creative Commons Atribución-NoComercial-SinDerivar 3.0 Internacional. 


\section{Inovação e colaboração governamental para recuperar veículos roubados e furtados}

Government innovation and collaboration to recover robbed and stolen vehicles

Innovación y colaboración de gobiernos para recuperar vehículos robados e hurtados

Arthur Viana Guimarães

Polícia Militar do Estado de São Paulo: São Paulo, São

Paulo, BR, Brasil

art.hur.v@hotmail.com

Sandro Cabral

Insper, Brasil

sandroc2@insper.edu.br

Priscila Fernandes Ribeiro

Insper, Brasil

PriscilaFR1@insper.edu.br

Marcelo Marchesini da Costa

Insper, Brasil

marcelomc5@insper.edu.br
Redalyc: https://www.redalyc.org/articulo.oa? id $=351568433012$

Recepción: 30 Marzo 2021

Aprobación: 18 Mayo 2021

Publicación: 01 Octubre 2021

\section{Resumo:}

Objetivo da pesquisa: $\mathrm{O}$ objetivo deste trabalho é discutir em que medida a intensidade de colaboração entre diferentes esferas governamentais molda o desempenho organizacional obtido a partir de inovações tecnológicas no setor público, tendo como base a implementação de um sistema de recuperação de veículos roubados e furtados em que a Prefeitura de São Paulo e a Polícia Militar do Estado de São Paulo agiram conjuntamente.

Enquadramento teórico: Utiliza-se como suporte para a análise a literatura sobre governança colaborativa e inovações em governos, com destaque para a sua aplicação na área de segurança pública.

Metodologia: Por meio de regressões logísticas e análise de clusters espaciais, analisamos dados referentes a 319.487 veículos subtraídos no município de São Paulo entre 2015 e 2017.

Resultados: Enquanto identificamos um aumento estatisticamente relevante na probabilidade de se recuperar um veículo em decorrência dos esforços colaborativos empregados, encontramos também resultados heterogêneos nos diferentes distritos do município de São Paulo.

Originalidade: Este trabalho supre uma deficiência da literatura na medida em que, apesar do crescente uso de inovaçóes como forma de aprimorar serviços públicos, pouco se sabe sobre a intensidade de colaboração necessária para gerar os resultados desejados diante da adoção de novas tecnologias.

Contribuições teóricas e práticas: Os resultados sugerem que a efetividade de inovações está associada à intensidade de colaboração entre os diferentes órgãos públicos. Isso indica caminhos para que a gestão pública potencialize os benefícios de inovações em suas atividades.

PalaVras-chaVe: Inovação, Colaboração, Segurança Pública.

\section{Abstract:}

Research goals: The purpose of this paper is to discuss the extent to which the intensity of collaboration between different government spheres shapes the organizational performance obtained from technological innovations in the public sector, based on the implementation of a system for recovering robbed and stolen vehicles, in which the Public Administration of the Municipality of São Paulo and the Military Police of the State of São Paulo acted together. 
Theoretical framework: The literature on collaborative governance and government innovation supports this analysis, with emphasis on its application in the area of public security.

Methodology: Through logistic regressions and analysis of spatial clusters, we analyzed data referring to 319,487 vehicles subtracted in the city of São Paulo between 2015 and 2017.

Results: While we identified a statistically significant increase in the probability of recovering a vehicle as a result of the collaborative efforts employed, we found also heterogeneous results in the different districts of the municipality of São Paulo.

Originality: This work fills a gap in the literature because, despite the increasing use of innovations as a way to improve public services, little is known about the intensity of collaboration necessary to generate the desired results in the face of the adoption of new technologies.

Theoretical and practical contributions: The results suggest that the effectiveness of innovations is associated with the intensity of collaboration between different public bodies. This indicates ways for public management to maximize the benefits of innovations in their activities.

KEYWORDS: Innovation, Collaboration, Public Security.

\section{ReSUMEN:}

Objetivo de la investigación: El objetivo de este trabajo es discutir en qué medida la intensidad de la colaboración entre diferentes ámbitos de gobierno configura el desempeño organizacional obtenido de las innovaciones tecnológicas en el sector público, a partir de la implementación de un sistema de recuperación de vehículos robados y hurtados, en el que actuaron conjuntamente el Municipio de São Paulo y la Policía Militar del Estado de São Paulo.

Marco teórico: La literatura sobre gobernanza colaborativa e innovaciones gubernamentales se utiliza como soporte para el análisis, con énfasis en su aplicación en el área de seguridad pública.

Metodología: Mediante regresiones logísticas y análisis de conglomerados espaciales, analizamos datos referentes a 319.487 vehículos sustraídos en la ciudad de São Paulo entre 2015 y 2017.

Resultados: Si bien identificamos un aumento estadísticamente significativo en la probabilidad de recuperar un vehículo como resultado de los esfuerzos colaborativos empleados, encontramos resultados heterogéneos en los diferentes distritos del municipio de São Paulo.

Originalidad: Este trabajo aporta una deficiencia en la literatura en el sentido de que, a pesar del uso creciente de innovaciones como forma de mejorar los servicios públicos, se conoce poco sobre la intensidad de la colaboración necesaria para generar los resultados deseados de la adopción de nuevas tecnologías.

Aportes teóricos y prácticos: Los resultados sugieren que la efectividad de las innovaciones está asociada a la intensidad de la colaboración entre diferentes organismos públicos. Esto indica formas para que la gestión pública maximice los beneficios de las innovaciones en sus actividades.

Palabras clave: Innovación, Colaboración, Seguridad Pública.

\section{INTRODUÇÃO}

Governos ao redor do mundo utilizam inovações para melhorar a qualidade dos serviços prestados à população (Cavalcante \& Cunha, 2017; Torfing, 2019). Inovações permitem integração entre diferentes esferas governamentais e políticas públicas capazes de beneficiar espectros mais amplos da população (Ribeiro, Diniz, de Oliveira, \& Faleiros, 2018). Na segurança pública, contexto deste trabalho, inovaçōes tecnológicas como bancos de dados informatizados para a identificação de criminosos (Bromberg, Charbonneau, \& Smith, 2019), inclusive pelo reconhecimento facial (Bradford, Yesberg, Jackson, \& Dawson, 2020), almejam melhorar a efetividade e a eficiência dos serviços prestados. Mesmo inovações com baixa complexidade na área de segurança, como a consulta de antecedentes criminais por meio de terminais embarcados em viaturas, requerem o envolvimento de diversos órgãos governamentais, demandando colaboração para que os resultados esperados sejam atingidos (Ansell \& Gash, 2008; Ingrams, 2017).

O objeto que se configura como problema desta pesquisa é a pouca clareza sobre qual intensidade de colaboração entre organizações governamentais é necessária para que uma inovação pública atinja os resultados desejados. Alguns autores afirmam que não é possível promover e sustentar inovações na gestão pública focando-se em uma organização isoladamente, tendo em vista a necessidade de atuação em rede, onde os diversos entes interagem com vistas à produção dos resultados esperados (Osborne \& Brown, 2011; 
Vickers, Lyon, Sepulveda, \& McMullin, 2017). Sabe-se também que a coprodução entre cidadãos e governo importa para o resultado obtido das inovações governamentais (Dos-Reis \& Isidro, 2019). Porém, mesmo havendo argumentos sobre a relação entre resultados de inovações em políticas públicas e colaboração entre gestores de diversos órgãos (Crosby, 't Hart, \& Torfing, 2017), a literatura da área de administração pública é silenciosa quanto aos efeitos da intensidade dessa colaboração. Dito de outra forma, pouco se sabe sobre como a intensidade de colaboração entre órgãos públicos afeta o desempenho observado em serviços públicos após a adoção de inovações. Por exemplo, não se sabe se a colaboração sempre melhora os resultados da inovação ou se há um nível mínimo de interação para que resultados positivos possam ser observados. Assim, o objetivo deste trabalho é discutir em que medida a intensidade de colaboração entre diferentes esferas governamentais molda o desempenho organizacional obtido a partir de inovações tecnológicas no setor público.

O trabalho avalia os resultados de uma nova tecnologia adotada pela Polícia Militar de São Paulo (PMESP) em conjunto com a Prefeitura de São Paulo para identificação de veículos furtados e roubados por radares de controle de tráfego, utilizando a tecnologia de Reconhecimento Ótico de Caracteres (OCR). A análise baseou-se na literatura de inovações no setor público (De Vries, Bekkers, \& Tummers, 2016; Luna-Reyes, Gil-Garcia, \& Romero, 2012; Osborne \& Brown, 2011; Pereira, Macadar, Luciano, \& Testa, 2017; Ribeiro et al., 2018) e de análises sobre a adoção de tecnologia por governos, sobretudo na área de segurança pública (Lima \& Miranda, 2013). Essa inovação identifica veículos por meio de câmeras de radares e notifica automaticamente as equipes policiais em caso de veículos subtraídos. A colaboração nessa experiência, no entanto, se restringe ao compartilhamento de dados das câmeras de trânsito, geridas pelo poder municipal, que permite integração desses dados com os sistemas informatizados da PMESP.

O trabalho utiliza dados extraídos do Sistema de Informações Operacionais da Polícia Militar (SIOPM), contendo todos os veículos subtraídos registrados de 2015 a 2017 na cidade de São Paulo. O SIOPM é um banco de dados criado pela PMESP que reúne informações detalhadas de todas as ocorrências registradas via chamadas de emergência (190), Disque Denúncia, ocorrências elaboradas via Boletim de Ocorrência Policial Militar e qualquer intervenção policial-militar realizada no estado. Ao estimar os resultados de uma nova tecnologia governamental por meio de dados observacionais, este trabalho demonstra o potencial de arranjos colaborativos inovadores para a criação de valor público (Lopez, Lopes, Souto, \& Sant'Ana, 2014; Pereira et al., 2017; Torfing, 2019) em áreas com fortes implicações sociais (Ribeiro et al., 2018).

Na próxima seção, analisa-se a literatura de inovações em governos, com foco em questões de segurança pública, e apresenta-se hipóteses específicas ao caso. Posteriormente, apresenta-se os procedimentos metodológicos adotados e a contextualização do caso (Sistema RADAR). Por fim, apresenta-se os resultados obtidos, discute-se suas implicações e identifica-se limitações da presente análise e sugestões para estudos futuros.

\section{REVISÃO TEÓRICA E HIPÓTESES: INOVAÇÕES E COLABORAÇÃO EM GOVERNOS}

Há diversos motivos para a inovação em governos. Entre os motivos mencionados na literatura estão a correção de falhas de mercado e de governo, a busca por soluções para um crescente volume de problemas públicos complexos e a demanda por melhores serviços públicos e mais participação nos processos governamentais (Cavalcante \& Cunha, 2017). Apesar do grande volume de pesquisas sobre inovações governamentais, ainda há limitações conceituais e empíricas nessa área, como o fato de boa parte dos estudos não definir o que vem a ser o construto inovação e da falta de análises quantitativas que testem os efeitos decorrentes da adoção de inovações no setor público (De Vries et al., 2016). A despeito disso, há esforços em formalizar o entendimento sobre inovação. Por exemplo, instituições internacionais, tais como a Organização para a Cooperação e o Desenvolvimento Econômico (OECD/Eurostat) (2018), caracterizam inovação como um produto ou processo novo ou melhorado em relação aos anteriormente utilizados por um determinado ator ou colocados à disposição aos seus clientes. 
Neste artigo, entende-se inovação como uma mudança de descontinuidade, o que permite distinguir entre inovação e mudanças organizacionais incrementais (Osborne \& Brown, 2011). Ao explicar essa diferenciação, Osborne e Brown (2011) argumentam que incrementos processuais se relacionam com habilidades existentes aos trabalhadores daquela organização, enquanto inovações requerem novas habilidades. Essas mudanças de descontinuidade, no entanto, podem ocorrer em diferentes níveis, como em processos, na governança de uma política, ou mesmo na concepção dessa ação (De Vries et al., 2016). Isso introduz a discussão sobre tipos de inovação. $\mathrm{Na}$ sistematização realizada por Cavalcante e Camões (2017) utiliza-se a tipificação da inovação em serviços, processos, processos tecnológicos e processos administrativos.

A descrição de inovações em governos frequentemente revela a existência de colaborações entre governos ou entre organizações públicas e privadas (OECD/Eurostat, 2018). Entende-se colaboração como uma forma de interação entre organizações (Poocharoen \& Ting, 2015), ainda que parte dos autores requeiram para tanto a tomada de decisão conjunta entre as partes (Thomson, Perry, \& Miller, 2009) enquanto outros autores reconhecem a possibilidade, sobretudo entre agências governamentais, de uma colaboração imposta ou determinada por regulamento (Hafer, 2018). Colaborações entre órgãos públicos podem ser caminhos para a alta performance de governos (Lazzarini, Pongeluppe, Ito, Oliveira, \& Ovanessoff, 2020). Dodgson (2014) afirma que colaboração é uma condição necessária para a inovação. Há diversos fatores que influenciam a decisão por inovar no setor público, sendo que a participação em redes e o relacionamento interorganizacional constam como fatores ambientais importantes para essa escolha, em conjunto com outros elementos de nível organizacional, individual e da própria característica da inovação em questão (Cavalcante \& Camões, 2017).

Um dos tópicos de interesse nessa literatura investiga o que determina o sucesso de inovações em governos. Nesse sentido, uma visão possível é que inovações em governos precisam compreender a complexidade da inovação e não simplesmente adotá-la pela novidade. Além disso, é necessário haver um mandato organizacional e político adequado para a adoção da inovação e gestores públicos precisam interagir em rede para criar as condições institucionais de sucesso para a inovação. Deve-se também adotar uma abordagem orientada ao cliente para a inovação em serviços públicos e, por fim, deve-se compreender o ambiente político em que se desenvolve a inovação em governos (Osborne \& Brown, 2011). Ou seja, o aspecto de rede e de compreensão do ambiente, explícitos na definição anterior, sugerem que arranjos colaborativos contribuam para o sucesso de inovações. Isso fica ainda mais explícito em autores que defendem a colaboração entre diferentes atores governamentais como necessária para mobilizar recursos e objetivos comuns (Vickers et al., 2017). Crosby e colaboradores (2017) defendem que a liderança da adoção de inovação em governos aja como um maestro de colaborações, e não como um herói inovador e solitário, dado o propósito de se gerar valor público. Nem todas as definições, no entanto, pontuam a necessidade de colaboração para o sucesso de inovações em governos. Determinados estudos enfatizam a necessidade de recursos organizacionais disponíveis, habilidades de liderança, disposição de se aceitar determinado risco decorrente da inovação, além de estruturas organizacionais ajustadas e incentivos adequados (De Vries et al., 2016).

Para verificar se colaboração é ou não condição para o sucesso de inovação na gestão pública, é preciso compreender o conceito de colaboração utilizado. Entre as muitas definições de colaboração em governos utilizadas na literatura, há um contínuo de relações intraorganizacionais progressivamente mais intensas, partindo de compartilhamento de informações, passando por partilha de recursos, combinação de atividades e chegando até o desenvolvimento de capacidades em comum para se atingir resultados que não poderiam ser atingidos pelas organizações em separado (Bryson, Crosby, \& Stone, 2015). A compreensão de que arranjos colaborativos são necessários para lidar com problemas públicos complexos gerou um crescente interesse na literatura sobre governança colaborativa, envolvendo órgãos públicos e seus parceiros privados (Ansell \& Gash, 2008; Bryson et al., 2015). Analisando colaboração entre agências governamentais, Nylén (2007) afirma que diversas combinações de intensidade e graus de formalização da parceria levam a distintos 
resultados, sendo o alto grau de formalização e alta intensidade de colaboração o arranjo ao mesmo tempo mais promissor e também mais instável.

Inovar para resolver problemas públicos complexos gera uma grande proximidade dos estudos sobre inovação e sobre arranjos de governança colaborativa (Torfing, 2019). Crosby e colaboradores (2017) propõem uma mudança de paradigma no setor público em que a inovação se combine com colaboração e interdependência para substituir a lógica procedimental e de comando e controle ou competição. Pressupõese que interações são importantes para inovação e que, portanto, colaboração entre órgãos públicos seja sempre relevante para ao sucesso da inovação em governos. Há também quem faça a relação entre inovação e co-criação, propondo que arenas de interação entre diversos atores organizacionais podem estimular a adoção de inovações (Dos-Reis \& Isidro, 2019; Torfing, Sørensen, \& Røiseland, 2019). Entretanto, em muitos casos ainda é possível, mesmo na gestão pública, caracterizar um problema na perspectiva exclusiva ou prioritária de uma única organização pública. De fato, a própria literatura de inovação em governos trata sobre diferentes tipos de inovação e propõe que inovações podem ocorrer em processos - administrativos ou tecnológicos - e também podem focar em produtos, serviços, governança ou mesmo no conceito de uma determinada política pública (De Vries et al., 2016).

Ainda que as observações até aqui tenham considerado o governo como um todo, antes da apresentação das hipóteses é importante um olhar específico sobre a inovação na atividade policial.

\section{Inovação na Ação Policial}

A atividade policial é composta por funções diversas, que incluem, por exemplo, procedimentos administrativos, patrulhamento e ações de enfrentamento. Estudos indicam que há grande discricionariedade dos policiais na escolha sobre quanto tempo dedicar a essas diferentes atividades (Wu \& Lum, 2017). A introdução de inovações, nesse sentido, é importante por viabilizar a automação de processos tediosos e propensos a distrações realizados diuturnamente por policiais (Mastrobuoni, 2020; Roberts \& Casanova, 2012). Ou seja, a inovação pode auxiliar no direcionamento mais efetivo de policiais às atividades que levam ao resultado desejado por sua organização.

As evidências, no entanto, não são uniformes no sentido de demonstrar que inovações melhoram a performance de organizações policiais (Lum, Koper, \& Willis, 2017). Apesar disso, o uso de novas tecnologias vem sendo positivamente associado ao aumento na capacidade de detecção de veículos subtraídos (Ozer, 2016). Ainda assim, é necessário cautela na promoção de inovações que podem reforçar distinções préexistentes, como entre policiais que exercem atividades de rua e os que exercem atividades administrativas (Willis, Koper, \& Lum, 2018).

Especificamente, a tecnologia auxilia a atividade policial reduzindo incertezas e permitindo que policiais exerçam a discricionariedade em uma abordagem, por exemplo, com maior segurança (Lum, Koper, \& Willis, 2017). Em estudos prévios constatou-se que o objetivo principal dos policiais se relaciona com maior eficiência que a inovação tecnológica pode propiciar, porém, tal visão pode dificultar outros tipos de inovação em processos ou governança, como uma abordagem de solução de problemas em sua origem ou de policiamento comunitário (Lum et al., 2017). Sendo assim, é necessário retomar a questão sobre qual é de fato o resultado do uso de inovações por forças policiais.

\section{Efetividade da Inovação}

Inovações em governos e mais especificamente em atividades policiais têm recebido atenção crescente nas últimas décadas (Ingrams, 2017; Luna-Reyes et al., 2012), inclusive no Brasil (de Moraes Sousa, Ferreira, 
Najberg, Medeiros, 2015; Pereira et al., 2017; Ribeiro et al., 2018), justamente por sua capacidade de aprimorar os serviços públicos prestados (Helbig, Gil-García, \& Ferro, 2009).

Para Janowski (2015), a adoção de tecnologias em governos segue uma evolução em quatro estágios, iniciando-se pela digitalização de processos, sem alteração na lógica de funcionamento organizacional, seguida por alteração nos processos e indo além, promovendo engajamento e finalmente contextualização, quando se modificam respectivamente a governança e o direcionamento de toda a estrutura de tecnologia em governo, visando ganhos de eficiência, efetividade ou simplificação de tarefa. Essas etapas poderiam envolver colaboração com parceiros, mas também podem ser medidas tomadas na organização isoladamente. Iniciativas para transformar governos tradicionais em "governos eletrônicos" possuem o potencial para alterar processos e práticas de uma organização - ou da interação entre organizações - visando ganhos de eficiência, efetividade ou simplificação de tarefas (Diniz, Barbosa, Junqueira, \& Prado, 2009).

Fundamentalmente, deseja-se investigar nessa primeira hipótese se a inovação com um mínimo de colaboração - isto é, com o apenas o compartilhamento de dados - é capaz de aprimorar os resultados obtidos por uma organização pública, dado que a literatura sobre governança tem se preocupado analisar os diferentes níveis de colaboração possíveis (Bryson et al., 2015). Deriva-se assim a primeira hipótese:

Hipótese $\mathbf{H}_{1}$ : A implementação de inovações tecnológicas está associada à melhoria de desempenho no setor público, ainda que com baixa intensidade de colaboração interorganizacional entre as organizações participantes.

\section{Heterogeneidade nos Resultados}

Como expresso anteriormente, parte da literatura relaciona o sucesso da inovação em governos a mudanças na estrutura de governança à promoção de maior colaboração entre diferentes órgãos (Hartley, Sørensen, \& Torfing, 2013; Vickers et al., 2017). Devido a limites orçamentários e à necessidade de atender às expectativas sobre uma melhoria contínua dos serviços públicos prestados, governos frequentemente recorrem a parcerias para a adoção de novas tecnologias (Torfing, 2019). Há diversos autores que buscam explicar determinantes de performance em arranjos de governança colaborativa, explorando, por exemplo, o papel das lideranças envolvidas, o ambiente regulatório e os processos de comunicação envolvidos (Ansell \& Gash, 2008; Bryso n et al., 2015). A efetividade das parcerias estabelecidas ainda depende de fatores como o número de parceiros envolvidos, o grau de compartilhamento de poder entre os envolvidos e a maneira pela qual os arranjos são formalizados (Brown, O’Toole, \& Brudney, 1998). Entre os fatores apontados por gerar dificuldades especificamente para o compartilhamento de informações figuram a existência de diferentes mecanismos de controle e procedimentos, a baixa confiança pré-existente, a escassez de recursos organizacionais e preocupações sobre um mal uso das informações (Yang \& Maxwell, 2011).

Diante da inexistência dos fatores de sucesso estabelecidos acima, que dependem de uma colaboração mais intensa, e com a possibilidade de prevalecerem os determinantes de insucesso da colaboração, supõese que o resultado da inovação de processos sem ajuste de governança seria uma maior heterogeneidade no aproveitamento dos dados compartilhados. Ou seja, no caso de tecnologias voltadas à recuperação de veículos, em que os resultados dependem da articulação entre sistemas e ações tomadas por partes operando de forma interdependente, é esperado que haja heterogeneidade nos resultados em função da própria variabilidade dos recursos que podem ser empregados e até mesmo da própria atenção alocada pelos policiais à recuperação de veículos roubados ou furtados. Há variação em capacidades técnica e administrativas das diferentes unidades de forças policiais, sendo esse um dos fatores que pode dificultar o sucesso na implementação de sistemas de informação intergovernamentais (Ribeiro et al., 2018). Uma determinada unidade policial pode estar mais preocupada em reduzir os homicídios do que em recuperar veículos roubados. Ou seja: 
Hipótese $\mathrm{H}_{2}$ : A falta de processos centralizados de governança colaborativa para suportar a implementação de inovações tecnológicas no setor público está associada a uma maior heterogeneidade de desempenho observado

A seção seguinte descreve os métodos e dados utilizados.

\section{DADOS E MÉTODOS}

Esta seção esclarece a metodologia adotada no estudo. Inicialmente, detalha-se o contexto institucional do Sistema RADAR, objetivo desta análise. Em seguida, faz-se os esclarecimentos necessários sobre a base de dados utilizada. Por fim, descreve-se a estratégia empírica utilizada.

\section{Contexto Institucional: o Sistema RADAR}

As informações desta seção baseiam-se no descritivo da experiência do Sistema RADAR, premiada como destaque em inovação de serviços públicos pela 11ª edição do Prêmio Mário Covas (PMESP, 2015). Além disso, a experiência profissional de um dos autores viabiliza conhecimento direto sobre a implementação dessa experiência.

Entre 2013 e 2016, o Brasil registrou uma média de aproximadamente 500.000 veículos furtados ou roubados por ano em seu território (Fórum Brasileiro de Segurança Pública [FBSP], 2019). Considerando apenas a cidade de São Paulo, foram registrados 82.830 veículos subtraídos em 2016, ano de implantação da inovação aqui analisada (Secretaria de Segurança Pública, 2017).

Em meio a esse contexto, surge o Sistema RADAR, criado e gerido pelo Centro de Processamento de Dados da PMESP. O sistema vincula bancos de dados da própria Polícia Militar, da Polícia Civil, da Secretaria da Fazenda e do INFOSEG - banco de dados federal que concentra as informações de roubo e furto de veículos de todo o país e de veículos de países que fazem fronteira com o Brasil -, com dados das câmeras de trânsito em um sistema de reconhecimento automático de emplacamento, instaladas pelas prefeituras. Assim, as equipes policiais próximas ao local em que o veículo subtraído é detectado recebem um alerta para que possam efetuar o cerco e captura dos criminosos, bem como recuperar o veículo.

Na política aqui considerada, a colaboração entre polícia e poder público municipal ocorre por meio do compartilhamento de dados. A escolha dos locais de instalação das câmeras para identificação dos veículos, o número de câmeras, o ritmo de instalação e sua manutenção cabem às prefeituras e têm como foco a fiscalização das condições de trânsito nas vias públicas.

O programa utilizado no Sistema RADAR envia automaticamente alertas para os Terminais Móveis de Dados (TMD) integrados nas viaturas policiais e ao Centro de Operações da Polícia Militar (COPOM), onde um agente pode também enviar o alerta via rádio para os policiais militares que operem sem TMD como, por exemplo, policiais em motocicletas. Os alertas são distribuídos às equipes num raio determinado individualmente pela PMESP por cada radar e as distâncias variam de acordo com a região instalada com a intenção de que seu empenho seja mais eficiente. Regiões centrais e mais urbanizadas costumam ter um raio menor e regióes periféricas ou interioranas, um raio maior. O Sistema RADAR, em tese, amplia tanto a possibilidade de abordagem direta de veículos com restrições (furto, roubo, proprietário foragido da justiça ou veículo envolvido em atividade criminosa), quanto viabiliza a elaboração de processos de planejamento para investigação de crimes e identificação de padrões delituosos, em linha com as melhores práticas internacionais de combate ao crime (Lu, 2003).

Quando a operação é bem-sucedida, o veículo é recuperado, os infratores são presos e os objetos de interesse são encaminhados ao Distrito Policial (Polícia Civil) para deliberações de polícia judiciária. As informações 
também são registradas em auto próprio (denominado Boletim de Ocorrência da Polícia Militar) pelo policial militar atuante.

\section{Base de Dados}

Este estudo utiliza dados sobre veículos furtados, roubados e (ou) localizados extraídos do SIOPM, entre janeiro de 2015 a dezembro de 2017, trimestralmente, dentro do município de São Paulo, uma base de dados que é alimentada pelos Boletins de Ocorrência da Polícia Militar (BOPMs). As bases de dados obtidas internamente, bem como as suas autorizações de uso, estão disponíveis junto aos autores e podem ser compartilhadas mediante solicitação. Esse sistema dispõe ainda de informações operacionais da PMESP, tais como efetivos empregados e outras operações policiais. O quantitativo de radares em atividade foi observado por meio do Relatório Analítico de Alertas por Sistema e Faixa de Hora, elaborado automaticamente pelo Sistema RADAR através da plataforma do COPOM Online, sistema de apoio operacional da PMESP.

A implementação do Sistema RADAR no município de São Paulo iniciou-se em julho de 2016, quando os primeiros radares foram instalados, chegando a 800 radares em 93 distritos administrativos da cidade ao longo de 2017.

A escolha da cidade de São Paulo deve-se ao fato de esta ser a cidade com maior densidade de radares, maior concentração do efetivo policial e maior volume de ocorrências policiais, inclusive as relacionadas a furtos e roubos de veículos. Isso faz do município um laboratório adequado para testar as implicações da adoção de novas tecnologias voltadas ao combate ao crime.

Cada uma das 319.487 observações refere-se a um veículo subtraído e (ou) localizado na cidade entre 2015 e 2017. Para cada veículo observa-se sua placa e todas as suas características diferenciadas por variáveis categóricas, tais como cor, marca e tipo de veículo.

A variável dependente é uma variável categórica (dummy) que assume valor zero quando o veículo subtraído não foi recuperado e 1 quando o veículo foi recuperado. A variável independente Programa indica se o sistema RADAR estava ou não em funcionamento no momento da ocorrência.

As variáveis de controle foram definidas a partir de fatores que a experiência policial sugere serem relevantes para a aumentar as chances de recuperação de um veículo subtraído. Dessa forma, a partir de interações com policiais organizou-se um bloco de características dos veículos, contendo tipo e cor, sendo todas dummies que assumem valor um caso descrevam a característica referenciada, ou zero, caso contrário. Espera-se que veículos que se destacam por sua excepcionalidade, como uma cor chamativa, sejam mais facilmente identificados pela força policial. Já o bloco de fatores operacionais engloba informações diretamente relacionadas à atividade policial. A escolha da média de efetivo policial militar por região é pautada em estudos que corroboram a eficiência de patrulhas em locais de alta incidência criminal (Di Tella \& Schargrodsky, 2004). As variáveis de operações policiais correspondem à média da quantidade de iniciativas especiais voltadas ao combate ao crime realizadas no trimestre. A Operação Direção Segura é realizada em vias arteriais, próximas a áreas de casas noturnas e bares, sem horário fixo, objetivando propiciar maior segurança aos usuários do sistema viário com foco na coibição e fiscalização do uso de bebidas alcoólicas por condutores. A Operação de Trânsito é feita com foco na fiscalização de trânsito em grandes corredores, locais com maior incidência de infrações administrativas ou trechos perigosos. Como tais operações aumentam a presença policial nas ruas, espera-se que sua existência também tenha efeito positivo na recuperação de veículos roubados e furtados. Há ainda efeitos fixos de tempo, de forma de capturar fatores sazonais que podem influenciar na maior ou menor propensão à recuperação de veículos.

A Tabela 1 descreve cada uma das variáveis utilizadas no modelo, apresentando a cada variável sua quantidade de observações, média, desvio padrão, bem como seus valores observados de mínimo e máximo, além dos principais aspectos da distribuição, com os percentis 5\%, 25\%, mediana, $75 \%$ e $90 \%$. 
Tabela 1 - Estatísticas Descritivas

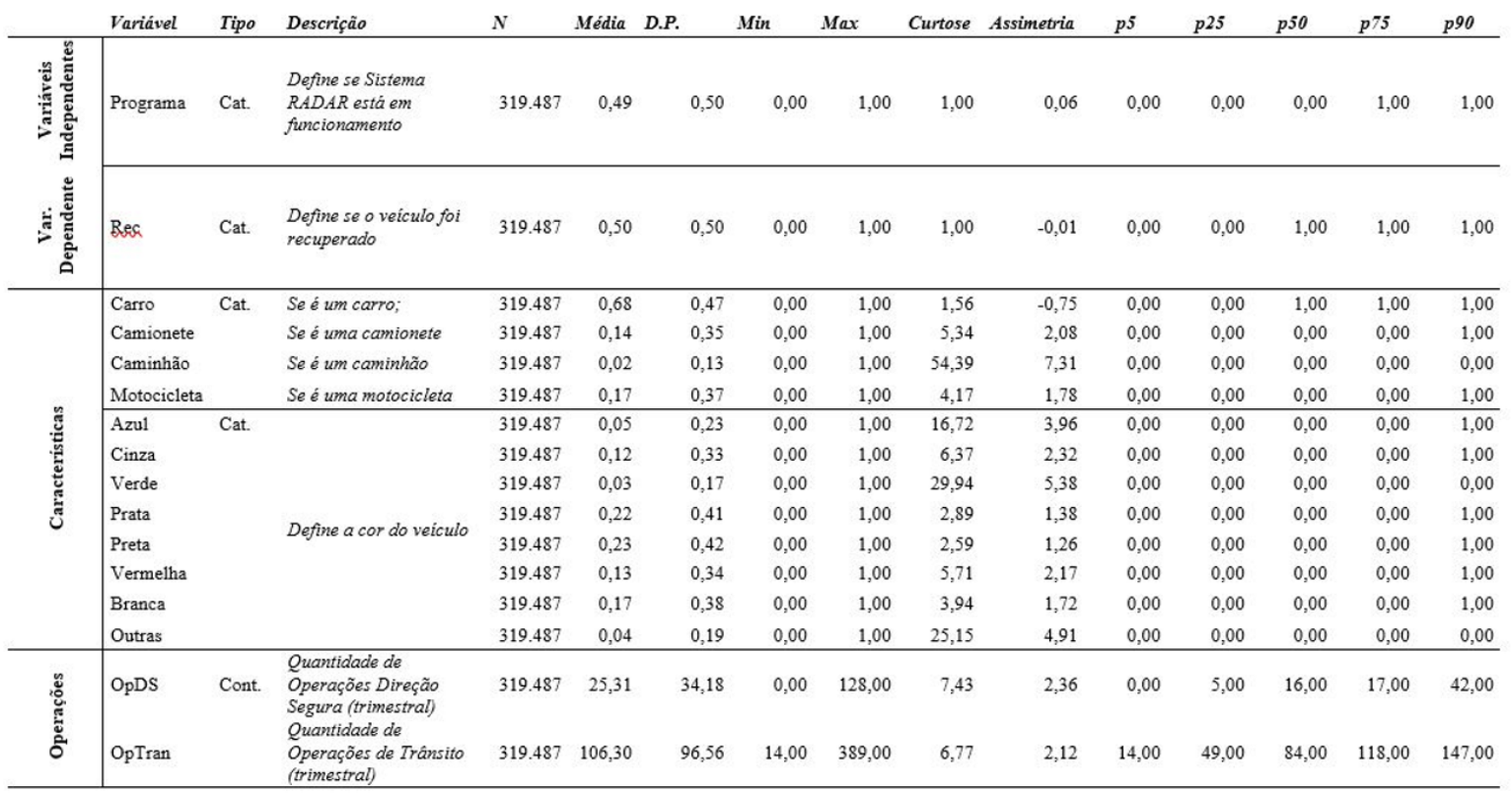

\begin{tabular}{|c|c|c|c|c|c|c|c|c|c|c|c|c|c|c|c|}
\hline & PMCentro & Cont. & $\begin{array}{l}\text { Média diária de } \\
\text { Policiais Militares em } \\
\text { serviço na região } \\
\text { central (trimestral) }\end{array}$ & 319.487 & 276,10 & 12,06 & 253,10 & 298,80 & 2,99 & $-0,32$ & 253,14 & 273,40 & 273,92 & 283,94 & 284,14 \\
\hline & PMNorte & & $\begin{array}{l}\text { Média diária de } \\
\text { Policiais Militares } \mathrm{em} \\
\text { na zona norte } \\
\text { (trimestral) }\end{array}$ & 319.487 & 346,10 & 11,52 & 331,10 & 373,40 & 3,42 & 0,86 & 331,06 & 339,06 & 345,06 & 352,34 & 356,59 \\
\hline & PMOeste & & $\begin{array}{l}\text { Média diária de } \\
\text { Policiais Militares em } \\
\text { serviço na zona oeste } \\
\text { (trimestral) }\end{array}$ & 319.487 & 286,30 & 10,62 & 273,40 & 307,40 & 2,53 & 0,78 & 273,40 & 280,40 & 284,89 & 293,42 & 304,72 \\
\hline & PMSul & & $\begin{array}{l}\text { Média diária de } \\
\text { Policiais Militares em } \\
\text { serviço na zona sul } \\
\text { (trimestral) }\end{array}$ & 319.487 & 721,40 & 18,47 & 693,70 & 759,40 & 2,51 & 0,45 & 693,71 & 701,98 & 723,11 & 731,38 & 745,30 \\
\hline \multirow[t]{4}{*}{ 育 } & Tri4 & Cat. & $\begin{array}{l}\text { Se a recuperação } \\
\text { ocorreu no } 4^{\circ} \text { Trimestre. }\end{array}$ & 319.487 & 0,25 & 0,43 & 0,00 & 1,00 & 2,34 & 1,16 & 0,00 & 0,00 & 0,00 & 0,00 & 1,00 \\
\hline & Ano2015 & \multirow[t]{3}{*}{ Cat. } & $\begin{array}{l}\text { Se a recuperação } \\
\text { ocorreu em } 2015\end{array}$ & 319.487 & 0,35 & 0,48 & 0,00 & 1,00 & 1,42 & 0,65 & 0,00 & 0,00 & 0,00 & 1,00 & 1,00 \\
\hline & Ano2016 & & $\begin{array}{l}\text { Se a recuperação } \\
\text { ocorreu em } 2016 .\end{array}$ & 319.487 & 0,34 & 0,47 & 0,00 & 1,00 & 1,45 & 0,67 & 0,00 & 0,00 & 0,00 & 1,00 & 1,00 \\
\hline & Ano2017 & & $\begin{array}{l}\text { Se a recuperação } \\
\text { ocorreu em } 2017 .\end{array}$ & 319.487 & 0,31 & 0,46 & 0,00 & 1,00 & 1,65 & 0,80 & 0,00 & 0,00 & 0,00 & 1,00 & 1,00 \\
\hline
\end{tabular}

Fonte: Elaborada pelos autores.

\section{Estratégia Empírica}

Foi definido um modelo-base para testar a primeira hipótese. A variável independente de interesse é uma dummy que assume valor 1 quando o programa está em funcionamento efetivo. O objetivo é identificar a mudança na probabilidade de se recuperar um veículo provocada pela variável de interesse (definidas como medida de performance do programa em teste), controlada pelas demais variáveis.

A variável explicada é a indicadora Recuperação.$R e c)$ e, conforme já explicado, Programa é a variável de interesse no modelo. O efeito da variável Programa foi controlado pelo tipo de veículo, cor, média diária de policiais militares em serviço no trimestre por região, operações e variações de tempo. O modelo pode ser representado pela seguinte equação: 


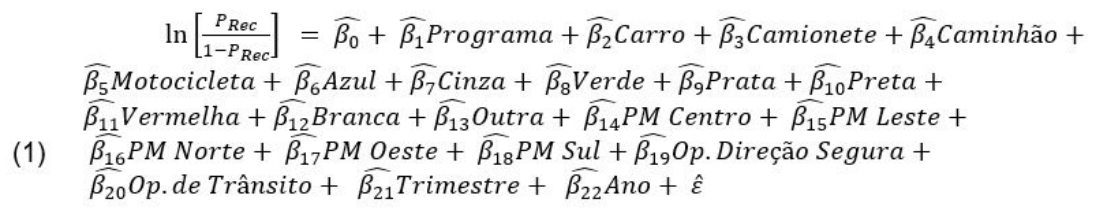

A equação representa o modelo de regressão logística, em que se estima o impacto de cada variável, na probabilidade de recuperação dos veículos,

Os modelos logit constituem um tipo particular de modelo com variáveis dependentes limitadas (Wooldridge, 2015). Ainda, como o logaritmo natural da razão de chances não nos permite verificar a mudança na probabilidade em sua magnitude, a análise será feita sob os efeitos marginais, ou seja, variações na probabilidade quando o preditor variar em uma unidade a partir de sua média.

Para testar a hipótese sobre a heterogeneidade dos resultados, realiza-se uma análise espacial de clusters dos veículos recuperados e promove-se a comparação com clusters dos veículos roubados ou furtados. A análise de clusters revela áreas com incidência de um determinado fenômeno acima ou abaixo do que seria esperado aleatoriamente em cada unidade de análise, indicando ainda como esse fenômeno se relaciona com as unidades vizinhas (Anselin \& Rey, 2014).

Para os testes econométricos aqui apresentados, utiliza-se o software Stata, enquanto as análises de clusters foram feitas no software GeoDa (Anselin \& Rey, 2014).

\section{RESULTADOS}

Essa seção apresenta os resultados para cada hipótese apresentada previamente. Em função das correlações detectadas, nem todos os controles foram incluídos simultaneamente nos modelos aqui apresentados. A matriz de correlações de Pearson pode ser disponibilizada pelos autores sob demanda.

\section{Efetividade da Tecnologia}

As estimações dos modelos são apresentadas em 2 partes, sendo a primeira apenas com as variáveis controle do bloco de características veiculares, tempo e variável de interesse Programa e a segunda contendo o bloco de características veiculares, fatores operacionais e a variável de interesse. Os coeficientes representam o efeito marginal na média. Ainda, vale ressaltar que a interpretação dos efeitos marginais de uma variável independente sobre a probabilidade de recuperação de um veículo subtraído é analisada como variação percentual nesta probabilidade.

Fundamentalmente, a variável Programa é estatisticamente significativa e positiva sempre que incluída. O efeito marginal sobre a probabilidade de recuperação veicular, pelos resultados obtidos, indica que a probabilidade de um veículo roubado ser recuperado devido à existência do Programa aumenta em 2\% controlando-se por tempo, características veiculares (Tabela 2 - Modelo 1).

As variáveis do bloco de características veiculares foram todas estatisticamente relevantes para explicar a recuperação com exceção da variável $V$ erde, relacionada à característica cor. Caminhões são os mais prováveis de serem recuperados (aumenta a probabilidade de se recuperar um veículo em 2,29\% com relação a carros) e motos os menos prováveis (diminui a mesma probabilidade em $5 \%$ com relação a carros), talvez por conta da alta mobilidade das motocicletas e baixa mobilidade dos caminhões. As cores prata e preta impactam negativamente a probabilidade de recuperação em 4,3\% e 3,4\%, respectivamente, tomando como referência a cor azul. Vale ressaltar que ambas as cores, juntas, somam $44 \%$ dos veículos na base de dados.

As operações Direção Segura e de Trânsito parecem ter baixa efetividade na probabilidade de recuperação de veículos. Além disso, a tendência de recuperação conforme a variação do efetivo policial médio não 
apresenta o comportamento positivo esperado em nenhum dos dois modelos. No modelo 2, o aumento do efetivo médio na região oeste impacta negativamente a recuperação veicular. Uma vez que o efetivo medido é o total de policiais em serviço por turno na região, não há como ter conhecimento sobre o emprego específico desses policiais na atividade operacional, bem como quantas equipes compóem patrulhamento a pé ou motorizado, o que pode explicar essa diferença. As variáveis de tempo foram removidas do modelo 2 devido à colinearidade com as variáveis de operaçóes, em especial o efetivo policial, indicando que esta variável pode capturar a dinâmica do tempo para explicar a propensão de recuperação de veículos.

O teste de Hosmer-Lemeshow testa a qualidade de ajuste nos modelos logits propostos, comparando as probabilidades ajustadas e as probabilidades observadas. As probabilidades previstas pelo modelo são ordenadas de modo crescente e, em seguida, separados em . grupos de tamanho aproximadamente iguais. Hosmer e Lemeshow (1980) propõem que seja utilizado, critério também adotado para avaliação de todos os modelos.

Dentre os modelos apresentados na Tabela 2, o modelo 2 foi o que apresentou, para qualquer nível de significância usual, a não rejeição de que o modelo seja adequado para explicação da recuperação do veículo. Tais resultados em conjunto, representados pelos indicadores de sensibilidade e especificidade, estão situados em níveis superiores a 50\%, reforçando, assim, a validade dos nossos achados.

Testamos ainda a efetividade do acréscimo de cada radar adicional ao sistema, obtendo resultados positivos e significativos. Não reportamos esses resultados em função das limitações de espaço, porém, os exercícios estão disponíveis sob requisição. 
Tabela 2 - Regressão logística

\begin{tabular}{|c|c|c|c|}
\hline & \multirow[b]{2}{*}{ Variáveis } & \multirow{2}{*}{$\frac{\text { (1) V.I. + C.V. + Tempo }}{\text { Efeito Marginal }}$} & \multirow{2}{*}{$\frac{\text { (2) V.I + C.V. + F.O }}{\text { Efeito Marginal }}$} \\
\hline & & & \\
\hline & Programa & $\begin{array}{l}0,0294^{* * *} \\
(0,00374)\end{array}$ & $\begin{array}{c}0,0145358^{* * * 8} \\
(0,0031738)\end{array}$ \\
\hline \multirow{22}{*}{ 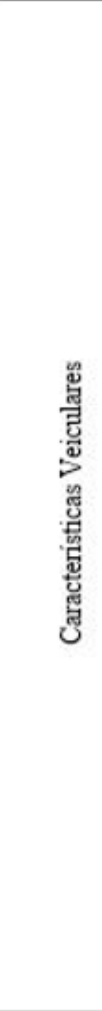 } & Carro & & \\
\hline & \multirow[t]{2}{*}{ Camionete } & $0,0121^{* * *}$ & $0,0120009 * * 8$ \\
\hline & & $(0,00274)$ & $(0,0027448)$ \\
\hline & \multirow[t]{2}{*}{ Caminhão } & $0,0229 * * *$ & $0,0230147^{* * 8}$ \\
\hline & & $(0,00687)$ & $(0,0068922)$ \\
\hline & \multirow[t]{2}{*}{ Motocicleta } & $-0,0500^{* * *}$ & $-0,0498817^{* * 8}$ \\
\hline & & $(0,00252)$ & $(0,0025363)$ \\
\hline & \multicolumn{3}{|l|}{ Azul } \\
\hline & \multirow[t]{2}{*}{ Cinza } & $-0,0108^{* *}$ & $-0,010799 * * *$ \\
\hline & & $(0,00463)$ & $(0,0046406)$ \\
\hline & \multirow[t]{2}{*}{ Verde } & $0,0104^{*}$ & 0,0103442 \\
\hline & & $(0,0063)$ & $(0,0063279)$ \\
\hline & \multirow[t]{2}{*}{ Prata } & $-0,0429 * * *$ & $-0,0429435^{* * 8}$ \\
\hline & & $(0,00432)$ & $(0,0043312)$ \\
\hline & \multirow[t]{2}{*}{ Preta } & $-0,0342^{* * *}$ & $-0,0342007^{* * *}$ \\
\hline & & $(0,00425)$ & $(0,0042549)$ \\
\hline & \multirow[t]{2}{*}{ Vermelha } & $-0,0154^{* * *}$ & $-0,015435^{* * *}$ \\
\hline & & $(0,00453)$ & $(0,00454)$ \\
\hline & \multirow[t]{2}{*}{ Branca } & $-0,00907 * *$ & $-0,009021^{* *}$ \\
\hline & & $(0,00448)$ & $(0,0044841)$ \\
\hline & \multirow[t]{2}{*}{ Outra } & $0,0110^{*}$ & $0,0109787^{*}$ \\
\hline & & $(0,00599)$ & $(0,0060101)$ \\
\hline \multirow{14}{*}{ 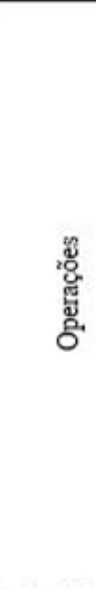 } & \multirow[t]{2}{*}{ PMCentro } & & 0,000432 \\
\hline & & & $(0,0002392)$ \\
\hline & \multirow[t]{2}{*}{ PMLeste } & & 0,0000677 \\
\hline & & & $(0,0000846)$ \\
\hline & \multirow[t]{2}{*}{ PMNorte } & & $0,0006375^{*}$ \\
\hline & & & $(0,0003619)$ \\
\hline & \multirow[t]{2}{*}{ PMOeste } & & $-0,001801^{* * *}$ \\
\hline & & & $(0,0001953)$ \\
\hline & \multirow[t]{2}{*}{ PMSul } & & $0,0007175^{* * 8}$ \\
\hline & & & $(0,0001461)$ \\
\hline & \multirow[t]{2}{*}{ Op. Direção Segura } & & $-0,0003162 * 8$ \\
\hline & & & $(0,0001416)$ \\
\hline & \multirow[t]{2}{*}{ Op. De Trânsito } & & $0,0000716^{*}$ \\
\hline & & & $(0,0000369)$ \\
\hline 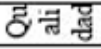 & Pseudo R-quadrado & 0,0026 & 0,0028 \\
\hline & Classificado Corretamente $(p>0,5)$ & $52,61 \%$ & $52,62 \%$ \\
\hline & Sensibilidade & $57,63 \%$ & $59,72 \%$ \\
\hline & Especificidade & $47,55 \%$ & $52,50 \%$ \\
\hline & Curva ROC (área) & 0,5344 & 0,5355 \\
\hline & p-valor Hosmer-Lemeshow (10 grupos) & $5,43 \%$ & $10,82 \%$ \\
\hline & Efeito Fixo de Tempo & Sim & Não \\
\hline & Obserações & 319.479 & 319.479 \\
\hline
\end{tabular}




\section{Heterogeneidade de Desempenho}

O número consolidado de roubos e furtos de veículos por distritos administrativos do município de São Paulo, no período entre 2015 e 2017 - representado no mapa "a" da figura 1 - apresenta poucos clusters. Todos os distritos em fundo cinza claro nos mapas não são o centro de um cluster. Ou seja, nesses distritos e em suas vizinhanças não se percebe uma distribuição do fenômeno diferente do que seria esperado aleatoriamente.

No entanto, existe um número maior de clusters de veículos recuperados do que dos veículos roubados ou furtados. Isso significa que, enquanto o roubo e furto de veículos parecem ser mais homogeneamente distribuídos pela cidade, existe maior heterogeneidade espacial na recuperação desses veículos, fornecendo indícios que suportam a segunda hipótese deste artigo. É preciso destacar, entretanto, que nesta análise não se pode atribuir a heterogeneidade especificamente ao Sistema RADAR. É possível que a recuperação de veículos já fosse concentrada em certas regióes e que após o Sistema RADAR a recuperação permaneça concentrada.

(a)

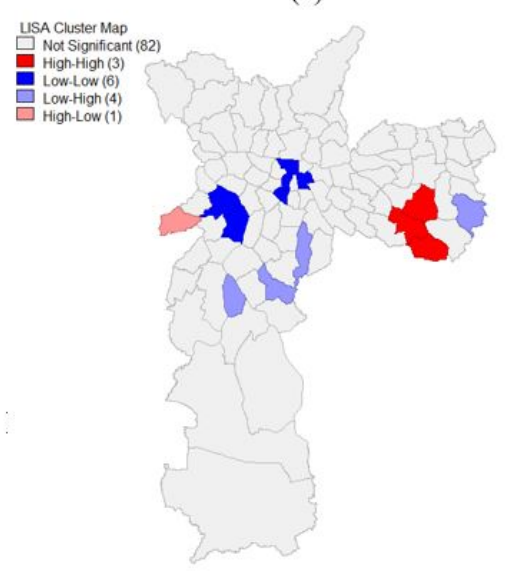

(b)

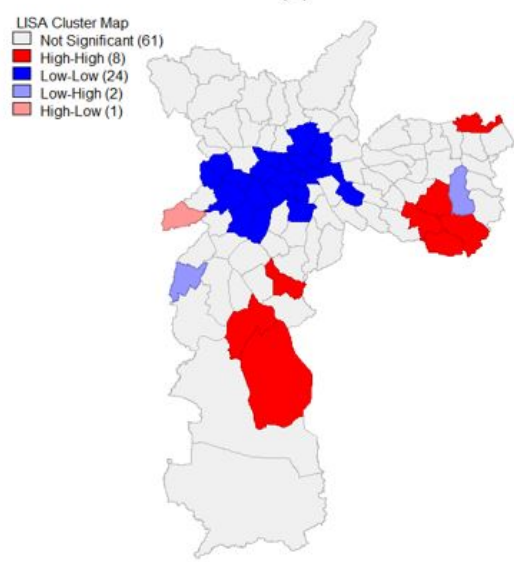

Figura 1 - Mapas de clusters de veículos roubados e furtados (a) e veículos recuperados pela Polícia Militar na cidade de São Paulo entre 2015 e 2017 (b)

Fonte: Elaborado pelos autores.

Especificamente nas zonas Leste e Sul observa-se a existência de distritos que são o centro de clusters HighHigh, onde a concentração do fenômeno naquela unidade é mais alta do que seria esperado aleatoriamente e circulada por unidades também com concentração mais alta do que seria esperado. Destaca-se que algumas regiões de grande concentração da recuperação de veículos são notórios pontos de desmanche e revenda de peças de veículos (Batista, 2016), o que facilita a ação policial de recuperação de veículos, independente do Sistema RADAR. Grande parte do centro expandido da cidade, por outro lado, apresenta clusters Low-Low, em que cada unidade e seus vizinhos apresentam uma menor concentração de veículos recuperados do que seria esperado aleatoriamente. É possível que essa menor recuperação de veículos nas áreas centrais seja devido a grandes pressões para o combate de outros tipos de crimes que podem receber maior atenção nessas áreas, tais como o roubo de pedestres e a necessidade de manter vigilância em pontos fixos para a manutenção da ordem pública, dificultando a abordagem a veículos subtraídos.

A análise de clusters permite ainda a identificação de duas áreas com características especiais. Os clusters Low-High são áreas de menor incidência de um fenômeno, circuladas por grande concentração desse 
fenômeno. No caso da recuperação de veículos, isso significa que algo pode estar fora do normal, como a ação de quadrilhas especializadas ou simplesmente má gestão policial, gerando resultado piores do que nas unidades vizinhas. Os clusters High-Low, por outro lado, são indicativos de unidades com grande concentração de um fenômeno circuladas por unidades com menos desse fenômeno do que seria esperado aleatoriamente. Nesse caso existe a oportunidade, por exemplo, de se identificar distritos em que as unidades policiais estejam fazendo melhor uso de informações e estratégias para a recuperação de veículos - como o Sistema RADAR, por exemplo. Ou seja, caso a PMESP e a Prefeitura de São Paulo optem por estreitar os laços de colaboração por meio de definição em conjunto dos locais de radares, planejamento de operações específicas e outras ações, há oportunidades para se aprimorar os resultados do sistema RADAR. Entre as diferentes unidades policiais os dados aqui apresentados evidenciam melhores práticas que podem ser replicadas. Maior intensidade na colaboração poderia levar a uma maior homogeneidade de resultados ao longo dos diferentes territórios.

\section{DISCUSSÃO E CONCLUSÃO}

Este trabalho se junta a um esforço pela realização de pesquisas que gerem informações úteis às gestoras e gestores públicos, com evidências robustas colhidas de bases administrativas governamentais (Isett, Head, \& VanLandingham, 2016; Parkhurst, 2017). O trabalho atende à demanda por uma maior compreensão dos determinantes de desempenho a partir de inovações tecnológicas no setor público, mais especificamente na área de segurança pública (Bromberg et al., 2019). Constatou-se um aumento na probabilidade de recuperação de veículos subtraídos após a adoção do Sistema RADAR.

Para além do simples efeito em termos de desempenho, esta pesquisa contribui para cimentar pontes entre as literaturas de colaboração intergovernamental (Agranoff \& McGuire, 2004; Ribeiro et al., 2018) e de inovações tecnológicas no setor público (De Vries et al., 2016; Luna-Reyes et al., 2012; de Moraes Sousa et al., 2015; Osborne \& Brown, 2011; Pereira et al., 2017; Ribeiro et al., 2018) ao demonstrar que inovações tecnológicas que alteram processos da atividade policial são capazes de aprimorar o resultado de serviços públicos, mesmo com um baixo grau de colaboração. Isso, porém, ocorre com grande heterogeneidade dos resultados em diferentes regióes. Ou seja, ter acesso a dados de outra agência governamental não levará sempre à mesma melhoria nos resultados. Isso ocorre na ausência de um esforço central de coordenação e governança entre as agências governamentais envolvidas. Nesse sentido, há oportunidades para uma inovação em governança capaz de amplificar os resultados alcançados.

Os resultados suportam as hipóteses de que a adoção de novas tecnologias e a intensidade do seu uso são efetivas na missão proposta: de aprimorar a recuperação veicular. A heterogeneidade nos resultados observados também suporta a hipótese de que, sem processos centralizados de governança colaborativa entre polícia e prefeitura, o objetivo primordial dos radares continua sendo o controle de tráfego e o uso das informações pelos diferentes batalhões policiais apresenta grande variação. Assim, se o objetivo for exclusivamente a melhoria de indicadores de desempenho estabelecidos para cada uma das organizações envolvidas, em linha com a inovação de processo, não é necessário estreitar a colaboração entre PMESP e Prefeitura de São Paulo para além do compartilhamento de dados. No entanto, se a intenção for tratar o roubo e furto de veículos como parte de problemas complexos da cidade, relacionados a outros fatores como a sensação de segurança, o desenvolvimento econômico e social da região, deve-se considerar uma inovação de governança, com uma colaboração entre órgãos públicos que inclua tomada de decisões e ações conjuntas. Ainda que alterar a governança seja uma possibilidade, a maior parte dos estudos sobre inovação em governos trata sobre novos processos (De Vries et al., 2016).

Por fim, nossos resultados não permitem afirmar que a introdução de inovações tecnológicas na ação policial substitua a experiência dos policiais. Há estudos indicando a relevância da experiência para se fazer 
um bom uso de evidências, juntando-se diversos conhecimentos necessários à atividade policial (Fleming \& Rhodes, 2018).

Assim, esta pesquisa traz implicações práticas e teóricas. Em termos práticos, demonstra-se que uma colaboração de baixa intensidade (Bryson et al., 2015), como uma rotina de compartilhamento de dados entre órgãos públicos, amparada por inovações tecnológicas, pode impulsionar mudanças capazes de beneficiar a população de forma mais ampla. Ou seja, uma inovação tecnológica é capaz de auxiliar gestores públicos (oficiais de polícia) a tomar decisões com base em evidências (Isett et al., 2016). Estudos do modus operandi de registros criminais combinados com informações do sistema implementado, por exemplo, podem indicar quadrilhas atuantes e fornecer informações para sua repressão ou captura. Assim, uma vez que programas dessa natureza dependem de convênios com municípios para que sejam expandidos, nossos resultados apesentam implicações práticas, na medida em que podem ajudar a tornar mais persuasivas as investidas dos órgãos de segurança pública em suas tratativas com as prefeituras, fomentando a adesão e empenho de recursos públicos no desenvolvimento e expansão de programas de colaboração intergovernamental mediados por plataformas tecnológicas.

Em termos teóricos, o estudo traz ponderações ao pressuposto implícito em parte da literatura sobre governança colaborativa, de que melhores resultados para serviços públicos dependem de complexas estruturas e processos de colaboração (Bryson et al., 2015). O estudo indica, por meio de resultados díspares em distintas regiões da cidade de São Paulo, que na ausência de colaboração mais intensa não há uma ressignificação dos indicadores e prioridades das organizações envolvidas. A PMESP faz uso dos dados disponíveis conforme as prioridades de cada região, ao passo que a recuperação de veículos roubados e furtados absolutamente não é um objetivo da Prefeitura de São Paulo, cujo foco é o controle de tráfego.

Entre as limitações do trabalho, não foi possível avaliar a relação custo-benefício do sistema RADAR por falta de acesso a dados, como o custo de manutenção das câmeras pelas prefeituras conveniadas. A falta de maiores informações sobre os veículos, tais como estar ou não sob cobertura de seguros e seu preço médio poderiam trazer mais informações relevantes ao estudo. Apesar de a atividade de polícia administrativa ser primariamente da Polícia Militar, a ausência de dados das atividades e recursos de outras forças policiais também são fatores importantes.

Futuros estudos podem abordar as limitações acima e promover coletas de informações mais detalhadas das forças policiais, testando sua relevância na recuperação veicular. Por fim, experimentos aleatorizados, considerando a densidade de radares em cada microrregião, também podem trazer informaçóes valiosas sobre o comportamento da atividade criminosa e sua relação com a malha viária, ajudando pesquisadores interessados nas relações entre novas tecnologias e seus impactos na criação de valor público a compreender melhor os mecanismos envolvidos.

\section{REFERENNCIAS}

Agranoff, R., McGuire, M.. (2004). Collaborative Public Management: New Strategies for Local Governments. Georgetown University Press.

Anselin, L., Rey, S. (2014). Modern Spatial Econometrics in Practice: A Guide to GeoDa, GeoDaSpace and PySAL. GeoDa Press LLC.

Ansell, C., Gash, A. 2008. "Collaborative Governance in Theory and Practice." Journal of Public Administration Research and Theory 18(4):543-71.

Batista, L. G. (2016). "O Negócio Que Mais Cresce: Notas Etnográficas Sobre o Mercado Do Roubo de Carros Em Uma Periferia de São Paulo.” Florestan (5):32-49.

Bradford, B., Yesberg, J., Jackson, J., Dawson, P. (2020). "Live Facial Recognition: Trust and Legitimacy as Predictors of Public Support for Police Use of New Technology." The British Journal of Criminology 60(6):1502-22. 
Bromberg, D. E., Charbonneau, É., Smith, A. (2019). "Public Support for Facial Recognition via Police Body-Worn Cameras: Findings from a List Experiment.” Government Information Quarterly 101415.

Brown, M. M., O'Toole, L. J. Jr., Brudney, J. L.. (1998). "Implementing Information Technology in Government: An Empirical Assessment of the Role of Local Partnerships." Journal of Public Administration Research and Theory $8(4): 499-526$.

Bryson, J. M., Crosby, B. C., Stone, M. M.. (2015). "Designing and Implementing Cross - sector Collaborations: Needed and Challenging." Public Administration Review 75(5):647-63.

Cavalcante, P. L. C., Camões, M. R. de S. (2017). "Inovação Pública No Brasil: Uma Visão Geral de Seus Tipos, Resultados e Indutores." in Inovação no setor público: teoria, tendências e casos no Brasil. Brasília: ENAP.

Cavalcante, P. L. C., Cunha, B. Q. (2017). “É Preciso Inovar No Governo, Mas Por Quê?” in Inovação no setor público: teoria, tendências e casos no Brasil. Brasília: ENAP.

Crosby, B. C., t'Hart, P., Torfing, Jacob. (2017). “Public Value Creation through Collaborative Innovation.” Public Management Review 19(5):655-69.

De Vries, H., Bekkers, V., Tummers, L. (2016). "Innovation in the Public Sector: A Systematic Review and Future Research Agenda." Public Administration 94(1):146-66.

Di Tella, R., Schargrodsky, E. (2004). “Do Police Reduce Crime? Estimates Using the Allocation of Police Forces after a Terrorist Attack.” American Economic Review 94(1):115-33.

Diniz, E. H., Barbosa, A. F., Junqueira, A. R. B., Prado, O. (2009). “O Governo Eletrônico No Brasil: Perspectiva Histórica a Partir de Um Modelo Estruturado de Análise." Revista de Administração Pública 43(1):23-48.

Dodgson, M. (2014). "Collaboration and Innovation Management." Pp. 462-81 in The Oxford Handbook of Innovation. Oxford: M. Dodgson, D.M. Gann, N. Philips.

Dos-Reis, M. C. A., Isidro, A. Filho. (2019). "Inovação Em Serviços e a Coprodução No Setor Público Federal Brasileiro." Administração Pública e Gestão Social.

FBSP. 2019. Crimes Patrimoniais - Tabela. Fórum Brasileiro de Segurança Pública.

Fleming, J., Rhodes, R. (2018). “Can Experience Be Evidence? Craft Knowledge and Evidence-Based Policing.” Policy \& Politics 46(1):3-26.

Hafer, J. A. 2018. "Understanding the Emergence and Persistence of Mandated Collaboration: A Policy Feedback Perspective of the United States's Model to Address Homelessness." The American Review of Public Administration 48(7):777-88.

Hartley, J., Sørensen, E., Torfing, J. (2013). “Collaborative Innovation: A Viable Alternative to Market Competition and Organizational Entrepreneurship." Public Administration Review 73(6):821-30.

Helbig, N., Gil-García, J. R., Ferro, E. (2009). "Understanding the Complexity of Electronic Government: Implications from the Digital Divide Literature." Government Information Quarterly 26(1):89-97.

Ingrams, A. (2017). "The Legal - normative Conditions of Police Transparency: A Configurational Approach to Open Data Adoption Using Qualitative Comparative Analysis." Public Administration 95(2):527-45.

Isett, K. R., Head, B. W., VanLandingham, G. (2016). "Caveat Emptor: What Do We Know about Public Administration Evidence and How Do We Know It?” Public Administration Review 76(1):20-23.

Lazzarini, S. G., Pongeluppe, L. S., Ito, N. C., Oliveira, F. de M., Ovanessoff, A. (2020). "Public Capacity, Plural Forms of Collaboration, and the Performance of Public Initiatives: A Configurational Approach.” Journal of Public Administration Research and Theory 30(4):579-95.

Lima, R. S. de, Miranda, Z. (2013). Nota Técnica: Política de Ciência, Tecnologia e Inovação Para a Segurança Pública. São Paulo: FBSP.

Lopez, F., Lopes, L. de F., Souto, B., Sant'Ana, D. (2014). “A Interação Entre Organizações Da Sociedade Civil e Governo Federal: Colaboração Nas Políticas Públicas e Aperfeiçoamentos Regulatórios Possíveis.” Pp. 329-46 in Brasil em Desenvolvimento 2014: Estado, Planejamento e Politicas Públicas. Brasília: IPEA.

Lu, Y. 2003. "Getting Away with the Stolen Vehicle: An Investigation of Journey-after-Crime." Professional Geographer. doi: 10.1111/0033-0124.5504002. 
Arthur Viana Guimarães, et al. Inovação e colaboração governamental para recuperar veículos roubad...

Lum, C., Koper, C. S., Willis, J. (2017). “Understanding the Limits of Technology's Impact on Police Effectiveness.” Police Quarterly 20(2):135-63.

Luna-Reyes, L. F., Gil-García, J. Romero, G.. 2012. “Towards a Multidimensional Model for Evaluating Electronic Government: Proposing a More Comprehensive and Integrative Perspective." Government Information Quarterly 29(3):324-34.

Mastrobuoni, G. (2020). “Crime Is Terribly Revealing: Information Technology and Police Productivity.” The Review of Economic Studies 87(6):2727-53.

de Moraes Sousa, M., Ferreira, V. da R. S., Najberg, E., Medeiros, J. J. (2015). "Portraying Innovation in the Public Service of Brazil: Frameworks, Systematization and Characterization." Revista de Administração 50(4):460-76.

Nylén, U. (2007). "Interagency Collaboration in Human Services: Impact of Formalization and Intensity on Effectiveness." Public Administration 85(1):143-66.

OECD/Eurostat. (2018). Oslo Manual 2018: Guidelines for Collecting, Reporting and Using Data on Innovation, 4th Edition, The Measurement of Scientific, Technological and Innovation Activities. Paris/Eurostat, Luxembourg.: OECD Publishing.

Osborne, S. P. Brown, L. (2011). “Innovation, Public Policy and Public Services Delivery in the UK. The Word That Would Be King?” Public Administration 89(4):1335-50.

Ozer, M. (2016). “Automatic Licence Plate Reader (ALPR) Technology: Is ALPR a Smart Choice in Policing?” The Police Journal 89(2):117-32. doi: 10.1177/0032258X16641334.

Parkhurst, J. (2017). The Politics of Evidence: From Evidence-Based Policy to the Good Governance of Evidence. Taylor \& Francis.

Pereira, G. V., Macadar, M. A., Luciano, E. M., Testa, M. G. (2017). "Delivering Public Value through Open Government Data Initiatives in a Smart City Context." Information Systems Frontiers 19(2):213-29.

PMESP. (2015). Prêmio Mário Covas 2015 - Relatório Descritivo Do Sistema RADAR. PMESP.

Poocharoen, O., Ting, B. (2015). “Collaboration, Co-Production, Networks: Convergence of Theories." Public Management Review 17(4):587-614.

Ribeiro, M. M., Diniz, E. H.; de Oliveira, L. C. P.; Faleiros, S. M. (2018). "Information Systems and Intergovernmental Relations in Brazilian Social Policies: A Study on Users' Adaptations to the Local Context." RAUSP Management Journal 53(1):86-97.

Roberts, D. J., Casanova, M. (2012). Automated License Plate Recognition (ALPR) Use by Law Enforcement: Policy and Operational Guide, Summary.

Secretaria de Segurança Pública. (2017). Ocorrências Registradas Por Mês. São Paulo.

Spanhol, F. J., Lunardi, G. M., de Souza, M. V. (2016). Tecnologias Da Informação e Comunicação Na Segurança Pública e Direitos Humanos. Editora Edgard Blücher.

Thomson, A. M., Perry, J. L., Miller, T. K. (2009). “Conceptualizing and Measuring Collaboration.” Journal of Public Administration Research and Theory 19(1):23-56.

Torfing, J. (2019). "Collaborative Innovation in the Public Sector: The Argument." Public Management Review 21(1):1-11.

Torfing, J., Sørensen, E., \& Røiseland, A. (2019). "Transforming the Public Sector into an Arena for Co-Creation: Barriers, Drivers, Benefits, and Ways Forward.” Administration \& Society 51(5):795-825.

Vickers, I., Lyon, F., Sepulveda, L., McMullin, C. (2017). “Public Service Innovation and Multiple Institutional Logics: The Case of Hybrid Social Enterprise Providers of Health and Wellbeing." Research Policy 46(10):1755-68.

Willis, J. J., Koper, C. S., Lum, C. (2018). "Technology Use and Constituting Structures: Accounting for the Consequences of Information Technology on Police Organisational Change." Policing and Society 1-19.

Wooldridge, J. M. (2015). Introductory Econometrics: A Modern Approach. Cengage learning.

Wu, X., \& Lum, C. (2017). "Measuring the Spatial and Temporal Patterns of Police Proactivity." Journal of Quantitative Criminology 33(4):915-34. 
Administração Pública e Gestão Social, 2021, vol. 13, núm. 4, OCtubre-Diciembre, ISSN: 2175-5787

Yang, T., Maxwell, T. A. (2011). "Information-Sharing in Public Organizations: A Literature Review of Interpersonal, Intra-Organizational and Inter-Organizational Success Factors." Government Information Quarterly 28(2):164-75. 